\title{
¿Son reversibles los efectos neurotóxicos de las drogas de síntesis?
}

\author{
EmiLlo Ambrosio \\ Catedrático de Psicobiología \\ Departamento de Psicobiología \\ Universidad Nacional de Educación a Distancia (UNED)
}

Enviar correspondencia a:

Emilio Ambrosio. Departamento Psicobiología (UNED). C/ Juan del Rosal, 10. Ciudad Universitaria 28040 Madrid.Teléfono: $91-398$ 79 73. Fax: $91-398$ 62 87 Correo electrónico: eambrosio@psi.uned.es

\section{RESUMEN}

La investigación preclínica sobre las drogas de síntesis realizada en animales de laboratorio nos indica que las acciones neurotóxicas pueden ser de dos tipos: a corto plazo y reversibles y a largo plazo e irreversibles. En efecto su consumo se traduce enseguida en un aumento de las concentraciones extracelulares de serotonina y dopamina que parece conllevar una subida de la temperatura corporal, pero este efecto es reversible en un tiempo medio de 6 horas. Sin embargo, una segunda acción aparece entre un día y una semana más tarde, con consecuencias neurotóxicas que afectan sobre todo al sistema serotoninérgico y a las terminaciones finas de las fibras nerviosas de serotonina que proceden de los núcleos del rafe dorsal, puede que sea por un incremento en la oxidación de ciertos procesos celulares en esas neuronas.

La suspensión de la administración de esas drogas hace que, a largo plazo, haya un nuevo crecimiento de las dañadas terminaciones finas, pero con un patrón de reinervación distinto al que originalmente había. Es probable que en humanos ocurra un proceso parecido, puesto que esto ocurren en primates no humanos. Tampoco conocemos las consecuencias psicológicas a largo plazo del daño neurotóxico que causan las drogas de síntesis en humanos incluida, en su caso, una posible reinervación de las terminaciones serotoninérgicas, si se abandona durante un tiempo considerable el consumo. Pero es sabido que la serotonina es un neurotransmisor implicado en números procesos cognitivos y emocionales y puede que sean la causa de las disfunciones psicológicas mantenidas que muestran los que han consumido drogas de síntesis.

Palabras clave: Neurotoxicidad, serotonina, dopamina, MDMA, MDA, metanfetamina, derivados de anfetamina, neurodegeneración, reinervación

\section{ABSTRACT}

It is clear from animal studies that substituted amphetamines have neurotoxic effects. Preclinical works have shown that amphetamine derivatives have two sort of effects: immediate and reversible, and prolonged and irreversible. The immediate effect of substituted amphetamine intake is an increment of extracellular levels of serotonin and dopamine that return to normality in about $6 \mathrm{~h}$ and may produce an augmentation of corporal temperature. However, this reversible effect is followed by other long lasting that usually occurs in the period between $24 \mathrm{~h}$ and one week. In this case, damage in serotonergic neurons appears, involving reuptake proteins and fine axon terminals, that are persistently lost. The mechanisms that might cause these neurotoxic effects are unknown, but it seems that an increment in cellular oxidative systems may have a role. Animal studies have also shown that an enduring cessation of amphetamine derivatives intake results in a progressive serotonergic re-innervation, but with a pattern that is different from the previously established during the normal development. Although we don't know if such re-innervation process may develop in human beings, is tempting to speculate that it will occur given that it happens in non-human primates. The behavioural consequences of serotonergic system damage and the hypothetical re-innervation process in humans are also unknown. However, they might be related to several behavioural malfunctions such as anxiety, depression or memory impairments that former amphetamine derivatives users exhibit in the span of their lives.

Key words: Neurotoxicity, serotonin, dopamine, MDMA, MDA, methamphetamine, substituted amphetamines, amphetamine derivatives, neurodegeneration, reinnervation. 
E n las sociedades desarrolladas actuales son millones las personas que en situaciones de ocio y diversión consumen drogas de síntesis habitualmente. Suelen hacerlo porque estas sustancias aumentan la empatía y despiertan un deseo de estar y/o hablar con otra gente en un estado emocional de cercanía que no se da con otras drogas químicamente similares, como los psicoestimulantes o los alucinógenos (Peroutka, Newman, y Harris, 1988). Se sabe que, además de esos efectos psicoactivos, las drogas de síntesis producen otros neurotóxicos y se ha planteado por ello la cuestión de si es o no su neurotoxicidad irreversible. A este respecto, en el estado actual de los conocimientos podemos decir que a grosso modo, los efectos de las drogas de síntesis parecen realizarse en dos fases: una inmediata y reversible y, otra, a largo plazo e irreversible. Con metodologías modernas como la microdiálisis intracerebral (un procedimiento que permite averiguar el tipo de neurotransmisores y las concentraciones en que se encuentran en el líquido extracelular que rodea a las neuronas) se ha comprobado que un efecto inmediato de las drogas de síntesis es el de liberar serotonina y dopamina en el encéfalo de roedores de laboratorio (Gough, Ali, Slikker, Holson, 1991); (Brodkin, Malyala, Nash, 1993). Además, se inhibe la recaptación de estos dos neurotransmisores (Steele, Nichols, Yim, 1987) y la actividad de la enzima MAO-A, que los degrada (Leonardi, Azmitia, 1994). El resultado global de estas acciones es que hay un aumento de la concentración extracelular de serotonina y dopamina, lo cual conlleva también un aumento de la temperatura corporal (Colado, Murray, Green, 1993). Estos efectos son reversibles puesto que duran menos de 6 horas, tiempo en el que las concentraciones extracelulares de esos neurotransmisores vuelven a la normalidad después de la administración de las drogas (Gudelsky y Nash, 1996; Mechán, Esteban, O'Shea, Eliot, Colado, Green, 2002).

Una segunda fase, neurotóxica, aparece entre 24 horas y una semana después de la administración de las sustancias. Durante ese período se produce una disminución de los niveles cerebrales de serotonina, asociada con una disminución funcional de los sitios de recaptación de este neurotransmisor, lo cual sugiere la existencia de daño neuronal. Estas alteraciones son diferentes según la especie animal de laboratorio estudiada. Así, en la rata y en el mono el daño se circunscribe al sistema serotoninérgico, mientras que en el ratón el sistema afectado es el dopaminérgico. Concretamente, se ha comprobado que el tratamiento con MDMA (éxtasis; metilendioximetanfetamina) en el ratón influye sobre las neuronas dopaminérgicas de la sustancia negra que proyectan al cuerpo estriado, resultando en una disminución de la concentración de dopamina en el cuerpo estriado, así como de sus metabolitos DOPAC y HVA (Logan, Alberti, Sanderson, Yee, 1988; O'Callagan y Miller, 1994; O'Shea,
Esteban, Camarero, Green, Colado, 2001; Colado, Camarero, Mechán, Sánchez, Esteban, Elliot, Green, 2001).

No conocemos aún los mecanismos por los que, en última instancia, se causa el daño neurotóxico en el sistema serotoninérgico en ratas de laboratorio y en primates no humanos. Sin embargo, se pueden apuntar algunos factores. Así, cuando se administra MDMA, uno de los primeros fenómenos que ocurren es la disminución de la actividad de la enzima triptófano hidroxilasa (TPH; la enzima que interviene en la síntesis de la serotonina) promovida posiblemente por la oxidación de esa enzima (Stone, Johson, Hanson, Gibb, 1989). Este proceso de oxidación de la TPH puede interferirse si se administran previamente inhibidores de la recaptación de serotonina (Schmidt y Taylor, 1990) o agentes antioxidantes como la cisteína (Steranka y Rhind, 1987). Sabemos, por otra parte, que uno de los agentes oxidantes celulares más conocidos son los radicales hidroxilo. Este tipo de radicales libres aumenta en el hipocampo como consecuencia de la administración de MDMA y se ha comprobado que cuando se administran sustancias que atrapan a esos radicales hidroxilo, como es el $\alpha$-fenil-N-ter-butilnitrona (PBN), el daño neurotóxico es menor (Colado y Green, 1995; Colado, O’Shea, Granados, Murria, Green, (1997).

Parece, por tanto, que una de las primeras acciones de drogas de síntesis como la MDMA sea la disminución o inactivación de los sistemas antioxidantes celulares. A su vez, la afectación de estos sistemas antioxidantes celulares da la impresión de ser un primer efecto neurotóxico temprano. Hay otros acontecimientos celulares más tardíos que parecen influir en la neurodegeneración de los terminales finos de serotonina. En estos procesos más tardíos parece que participa el neurotransmisor dopamina en un modo que depende de su grado de liberación, tras la administración de la droga. A este respecto, en general se puede establecer una graduación en la cantidad de dopamina liberada por las drogas de síntesis más comunes. Así, por ejemplo, la metanfetamina es muy efectiva en facilitar la liberación de serotonina, pero menos eficaz en la de dopamina. La MDA (metilendioxianfetamina) es una potente droga liberadora de serotonina, pero menos aún que la primera en liberar dopamina. A su vez, la MDMA es también eficaz en liberar serotonina, pero mucho menos que las dos drogas anteriores. $Y$, por último, la MDEA (metilendioxietilenanfetamina) es similar a la MDMA en la liberación de serotonina, pero mucho menos eficaz que todas las anteriores en la liberación de dopamina. Si, por otro lado, consideramos: 1) que todas las drogas citadas producen una disminución de la actividad de la enzima TPH en una fase temprana, y que 2) existe poca neurotoxicidad causada por la MDEA, puede pensarse que no se producirá daño neurotóxico en una fase tardía, si 
no se libera una determinada cantidad de dopamina. No obstante, se ha demostrado que la administración de L-DOPA (un precursor de la dopamina) junto con MDMA produce un aumento de los niveles extracelulares de dopamina, pero dicho aumento no conlleva una mayor neurotoxicidad una semana más tarde (Colado, O’Shea, Granados, Esteban, Martín, Green, 1999).

Las fibras nerviosas de serotonina que se dañan proceden de neuronas cuyos cuerpos celulares se ubican a lo largo del tronco del encéfalo agrupados en núcleos denominados núcleos del rafe. Hay dos grandes grupos de estos núcleos: los del rafe dorsal y los del rafe medial. De las fibras procedentes de estos dos tipos de núcleos, las que se dañan son las del rafe dorsal mientras que las del rafe medial son más resistentes (Molliver, Mamounas, Wilson, 1989). Al cabo de varios meses desde el tratamiento inicial con MDA o con MDMA se ha visto que, en animales, puede producirse una regeneración de las proyecciones serotoninérgicas dañadas provenientes del rafe dorsal. Sin embargo, el patrón de reinervación parece ser ligeramente distinto. Así, se ha observado en monos rhesus que, a los 12 meses después de haber finalizado el tratamiento con MDMA y, en consecuencia, de haberse producido el daño, la regeneración conlleva que haya un mayor crecimiento en el número de terminaciones finas en ciertas ramas colaterales de los axones y que crezcan ramas colaterales que antes no existían. En ratas, también se ha observado que a los 8 meses después del tratamiento con MDA hay una regeneración de las terminaciones finas de serotonina desde áreas de la corteza frontal hacia la occipital.

No tenemos medios técnicos actualmente para visualizar in vivo en humanos si se dan fenómenos similares en las terminaciones finas de serotonina, pero considerando que en monos y ratas la administración de drogas de síntesis disminuye la unión a las proteínas transportadoras que participan en la recaptación de serotonina y en humanos que han sido consumidores de MDMA se produce esa misma disminución (Semple, Ebmeier, Glabus, O'Carroll, Johnstone, 1999; Ricaurte, McCann, Szabo, Scheffel, 2000; Reneman, Booij, de Bruin, Reitsma, de Wolf, Gunning, den Heeten, van den Brink, 2001), es lógico hipotetizar que puede causarse un daño neurotóxico en esos terminales axónicos de humanos y, en su caso, una regeneración parecida a la que ocurre en primates de laboratorio. Las consecuencias comportamentales que pueda tener ese daño sobre las terminaciones finas de serotonina y su posterior regeneración con patrones diferentes a los previamente establecidos durante el normal desarrollo se desconocen. No obstante, dado que la serotonina es un neurotransmisor implicado en numeros procesos cognitivos y emocionales, puede que las disfunciones psicológicas persistentes que muestran los que han consumido drogas de síntesis, tales como ansiedad, depresión y deterioro de la memoria, estén relacionadas con la neurotoxicidad que les han causado estas sustancias y con la reorganización de los circuitos neurales que, posiblemente, es otra de las consecuencias negativas del daño duradero que producen las drogas de síntesis.

\section{REFERENCIAS}

Brodkin, J., Malyala, A., Nash, J.F. (1993). Effect of acute monoamine depletion on 3,4-methylenedioxymethamphetamine-induced neurotoxicity. Pharmacol. Biochem. Behav., 45, 647-653.

Colado, M.I., Murray, T.K., Green, A.R. (1993). Loss in rat brain following 3,4-methylenedioxymethamphetamine (MDMA), p-chloroamphetamine and flenfluramine administration and effects of chlormethiazole and dizocilpine. Br. J. Pharmacol., 108, 583-589.

Colado, M.I., Green., A. R. (1995). The spin trap reagent alfa-phenyl- $\mathrm{N}$-tert butil nitrone prevents 'ecstasy'induced neurodegeneration of \%-Ht neurones. Eur. J. Pharmacol., 280, 343-346.

Colado, M.I., O'Shea, E., Granados, R., Murria, T.K., Green, A.R. (1997). In vivo evidence for free radical involvement in the degeneration of rat brain $5-\mathrm{HT}$ neurones which follows administration of MDMA ('ecstasy'), but not the degeneration which follows flenfluramine. Br. J. Pharmacol., 121, 889-900.

Colado, M.I., O’Shea, E., Granados, R., Esteban, B., Martín, A.B., Green, A.R. (1999). Studies of the role of dopamine in the degeneration of 5-HT nerve endings in the brain of Dark Agouti rats following 3,4-methylenedioxymethamphetamine (MDMA or 'ecstasy') administration. Br. J. Pharmacol., 126, 911924.

Colado, M.I., Camarero, J., Mechán, A.O., Sánchez, V., Esteban, B., Elliot, J.M., Green, A.R. (2001). A study of the mechanisms involved in the neurotoxic action of 3,4-methylenedioxymethamphetamine on dopamine neurones in mouse brain. Br.J. Pharmacol., 134, 17111723.

Gough, B., Ali, S.F., Slikker, W., Holson, R.R. (1991). Acute effects of 3,4-methylenedioxymethamphetamine (MDMA) on monoamines in rat caudate. Pharmacol. Biochem. Behav., 39, 619-623.

Gudelsky, G.A., Nash, J.F. ( 1996). Carrier mediated release of serotonin by 3,4-methylenedioxymethamphetamine: implications for serotonin-dopamine interactions. J. Neurochem., 66,243-249.

Johnson, M.P., Conarty, P.F., Nichols, D.E. ( 1991). [3H]monoamine releasing and uptake inhibition properties of 3,4-methylenedioxymethamphetamine and pchloroamphetamine analogues. Eur. J. Pharmacol., 200, 9-16.

Leonardi, E.T., Azmitia, E.C. ( 1994). MDMA (Ecstasy) inhibition of MAO type $A$ and type $B$ : comparisons 
with fenfluramine and fluoxetine (Prozac). Neuropsychopharmacology, 10, 231-238.

Logan, B.J., Alberti, R., Sanderson, W.D., Yee, Y.B. (1988). Differences between rats and mice in MDMA (methylenedioxymethamphetamine) neurotoxicity. Eur. J. Pharmacol., 152, 227-234.

Mechán, A.O., Esteban, B., O'Shea, E., Elliot, J.M., Colado, M.I., Green, A.R. (2002). The pharmacology of the acute hyperthermic response that follows administration of 3,4-methylenedioxymethamphetamine (MDMA, 'ecstasy') to rats. Br. J. Pharmacol., 135, 170-180.

Molliver, M.E., Mamounas, L.A., Wilson, M.A. (1989). Effects of neurotoxic amphetamines on serotonergic neurons: immunocytochemical studies. In Pharmacology and Toxicology of Amphetamine and Related Designer Drugs. National Institute on Drug Abuse Research Monograph \# 94, 279-305.

O'Callagan, J.P., Miller, D.B. (1994). Neurotoxicity profiles of substituted amphetamines in the C57BL/6. J. J. Pharmacol. Exp. Ther., 270,741-751.

O'Shea, E., Esteban, B., Camarero, J., Green, A.R., Colado, M.I. (2001). Effect of GBR12909 and fluoxetine on the acute and long-term changes induced by MDMA, ('ecstasy') on the 5-HT and dopamine concentrations in mouse brain. Neuropharmacology, 40, 65-74.

Peroutka, S.J., Newman, H., Harris, H. (1988). Subjetive effects of 3,4-methylenedioxymethamphetamine in recreational users. Neuropsychopharmacology, 1, 273277.

Reneman, L., Booij, L., de Bruin, K., Reitsma, J. B., de Wolf, F. A., Gunning, W. B., den Heeten, G. J., van den Brink, W. (2001). Effects of dose, sex, and long- term abstention from use on toxic effects of MDMA (ecstasy) on brain serotonin neurons. Lancet, 358, 1864-1869.

Ricaurte, G.A., McCann, U.D., Szabo, Z., Scheffel, U. (2000). Toxicodynamics and long-term toxicity of the recreational drug, 3, 4-methylenedioxymethamphetamine (MDMA, 'Ecstasy'). Toxicol. Lett., 112-113, 143-146.

Schmidt, C.J. (1987). Neurotoxicity of the psychodelic amphetamine, methylenedioxymethamphetamine. J. Pharmacol. Exp. Ther., 240, 1-7.

Schmidt, C.J., Taylor, V.L. (1990). Reversal of the acute effects of 3,4-methylenedioxymethamphetamine by 5 HT uptake inhibitors. Eur. J. Phramacol., 181, 133-136.

Semple, D.M., Ebmeier, K.P., Glabus, M.F., O'Carroll, R.E., Johnstone, E.C. (1999). Reduced in vivo binding to the serotonin transporter in the cerebral cortex of MDMA ('ecstasy') users. Br. J. Psychiatry, 175, 63-69.

Steele, T.D., Nichols, D.F., Yim, G.K. (1987). Stereochemical effects of 3,4-methylenedioxymethamphetamine (MDMA) and related amphetamine derivatives on inhibition of uptake of [3H]-monoamines into synaptosomes from different regions of rat brain. Biochem. Pharmacol., 36, 297-303.

Steranka, L.R., Rhind, A. W. (1987). Effect of cysteine on the persistent depletion of brain monoamines by amphetamine, p-chloroamphetamine and MPTP. Eur. J. Pharmacol., 133, 191-197.

Stone, D.M., Johson, M., Hanson, G.R., Gibb, J.W. (1989) Acute inactivation of tryptophan hydroxylase by amphetamine analogs involves the oxidation of sulfhydryl sites. Eur. J. Pharmacol., 172, 93-97. 